Dear Author,

Please, note that changes made to the HTML content will be added to the article before publication, but are not reflected in this PDF.

Note also that this file should not be used for submitting corrections. 


\title{
Cell surface localised Hsp70 is a cancer specific regulator of clathrin-independent endocytosis
}

\author{
Benedikt Nimmervoll a , Lilia A. Chtcheglova a,b ${ }^{a}$ Kata Juhasz ${ }^{a}$, Nunilo Cremades ${ }^{c}$, Francesco A. Aprile ${ }^{c}$, \\ Alois Sonnleitner ${ }^{\mathrm{a}}$, Peter Hinterdorfer ${ }^{\mathrm{a}, \mathrm{b}}$, Laszlo Vigh ${ }^{\mathrm{d}}$, Johannes Preiner ${ }^{\mathrm{a}}$, Zsolt Balogi ${ }^{\mathrm{a}, \mathrm{e}, *}$ \\ ${ }^{a}$ Center for Advanced Bioanalysis GmbH, Linz, Austria \\ ${ }^{\mathrm{b}}$ Institute for Biophysics, Johannes Kepler University, Linz, Austria \\ ${ }^{\mathrm{C}}$ Department of Chemistry, University of Cambridge, Cambridge, United Kingdom \\ ${ }^{\mathrm{d}}$ Institute of Biochemistry, Biological Research Centre, Hungarian Academy of Sciences, Szeged, Hungary \\ ${ }^{\mathrm{e}}$ Institute of Experimental Medicine, Hungarian Academy of Sciences, Budapest, Hungary
}

\section{A R T I C L E I N F O}

\section{Article history:}

Received 3 May 2015

Revised 1 July 2015

Accepted 19 July 2015

Available online $\mathrm{xxxx}$

Edited by Lukas Huber

\section{Keywords:}

Hsp70

Endocytosis

Cancer

Membrane

Clustering

\begin{abstract}
A B S T R A C T
The stress inducible heat shock protein 70 (Hsp70) is present specifically on the tumour cell surface yet without a pro-tumour function revealed. We show here that cell surface localised Hsp70 (sHsp70) supports clathrin-independent endocytosis (CIE) in melanoma models. Remarkably, ability of Hsp70 to cluster on lipid rafts in vitro correlated with larger nano-domain sizes of sHsp70 in high sHsp70 expressing cell membranes. Interfering with Hsp70 oligomerisation impaired sHsp70-mediated facilitation of endocytosis. Altogether our findings suggest that a sub-fraction of sHsp70 co-localising with lipid rafts enhances CIE through oligomerisation and clustering. Targeting or utilising this tumour specific mechanism may represent an additional benefit for anti-cancer therapy.
\end{abstract}

๑) 2015 Federation of European Biochemical Societies. Published by Elsevier B.V. All rights reserved.

\section{Introduction}

Members of the Hsp70 family assist in the folding of nascent or misfolded proteins with an important role in cellular protein homeostasis [1]. Frequently upregulated in tumour cells [2], inducible Hsp70 (HSPA1A) exerts cytoprotective and anti-apoptotic functions [3]. Unlike the constitutive homologue Hsc70, Hsp70 is also known to be present at the extracellular leaflet of the plasma membrane (surface Hsp70, sHsp70) of most tumour cells but not that of the corresponding normal cells [4]. sHsp70 has been identified as a recognition feature for the immune system [5]. At the same time, the concentration of sHsp70 has been shown to correlate with metastatic potential of B16 mouse melanoma cells [6]. As surface localisation of Hsp70 is dependent on cholesterol content of the membrane, sHsp70 is likely associated with "lipid rafts" [7].

Author contributions: ZB, BN, JP conceived the study; BN, LAC, JP, ZB, KJ performed the experiments; BN, LAC, JP, ZB, LV analysed the data; AS, PH, ZB, NC, FAA provided reagents and tools; all authors discussed the results and wrote the paper.

* Corresponding author at: Institute of Experimental Medicine, Hungarian Academy of Sciences, Budapest, Hungary. Fax: +36 12109423.

E-mail addresses: balogi.zsolt@koki.mta.hu, zsolt.balogi@gmail.com (Z. Balogi).
Given that Hsp70 is frequently upregulated in tumour cells, where it is specifically present on the tumour cell surface, and that derailed endocytosis is a feature of cancer [8], we hypothesized that sHsp70 may have a general impact on the endocytic process. We previously showed that overexpression of Hsp70 in B16 mouse melanoma cells gave rise to the concentration of sHsp70 [9]. Here we show for the first time, using the B16 model and human A375 melanoma cells, that high levels of Hsp70 facilitate CIE even at unstressed conditions. We further reveal that a sub-fraction of sHsp70 localised in lipid rafts facilitates CIE through its oligomerisation and clustering in the plasma membrane.

\section{Materials and methods}

Inducible cell lines of B16-F10 and A375 (ATCC, Manassas, VA, USA) were generated by co-transfection of cells with pcDNA.6/TR and with empty pcDNA.4/TO or pcDNA.4/TO-mHSP70 or pcDNA.4/TO-mHsp70-E3. Stable clones were selected by media containing Zeocin (Life Technologies) and Blasticidin (InvivoGen, San Diego, CA, USA). B16-F10 cells were cultured in RPMI medium (Life Technologies) supplemented with 10\% FCS (Sigma-Aldrich, St. Louis, MO, USA), 2 mM L-glutamine (Life Technologies), A375 cells 
in DMEM/F12 (+L-glutamine) (Life Technologies) with 10\% FCS (Life Technologies). Hsp70 overexpression was induced by $2 \mu \mathrm{g} / \mathrm{ml}$ doxycycline for $16 \mathrm{~h}$, referred as "Hsp70". Hsp70 expression levels were tested by Western blotting and flow cytometry. Experiments with fluorescent readout were performed in OptiMEM supplemented with $1 \%$ FCS (Sigma).

To determine endocytic pathway activities, cells were incubated for $5 \mathrm{~min}$ at $37^{\circ} \mathrm{C}$ with $7.5 \mu \mathrm{M}$ FM4-64 (Sigma) or $1.5 \mu \mathrm{M}$ BODIPY-lactosylceramide (LacCer, Life Technologies) or $5 \mu \mathrm{g} / \mathrm{ml}$ transferrin-AlexaFluor488 (Tf, Life Technologies). Following the incubation, cells were washed twice in ice-cold medium. LacCer was back-exchanged in $6 \times 10$ min washing steps with $5 \%$ defatted BSA (Sigma) in ice-cold OptiMEM. Samples were analysed by flow cytometry (BD FACS Aria, Becton Dickinson, Franklin Lakes, NJ, USA) with an excitation at $488 \mathrm{~nm}$ or $633 \mathrm{~nm}$ and detection at $530 / 30 \mathrm{~nm}$ or at $695 / 40 \mathrm{~nm}$ for Tf and LacCer or for FM4-64, respectively. Debris and cells with damaged membranes were gated by FSC vs. SSC plotting and propidium-iodide exclusion ( $5 \mu \mathrm{g} / \mathrm{ml}$, Sigma). The extracellular Tf signal was quenched by $0.1 \%$ trypan blue (Sigma).

For the identification of sHsp70 endocytosis pathways, induced cells were pre-incubated for $30 \mathrm{~min}$ with $10 \mu \mathrm{g} / \mathrm{ml}$ chlorpromazine (Sigma) or $2 \mathrm{mM}$ amantadine (Sigma) for inhibition of CDE or with $50 \mu \mathrm{g} / \mathrm{ml}$ nystatin (Millipore) or $1 \mathrm{mM}$ methyl- $\beta$-cyclodextrin (M $\beta C D$, Sigma) for inhibition of CIE. Then, Alexa488 labelled (Life Technologies) cmHsp70.1 antibody (multimmune, München, Germany) or appropriate isotype control (Sigma) was added $(1 \mu \mathrm{g} / 50 \mu \mathrm{l})$ at $37^{\circ} \mathrm{C}$ for $30 \mathrm{~min}$ for endocytosis. Cells were washed in ice-cold medium and the intracellular fluorescence was determined by flow cytometry in the $530 / 30 \mathrm{~nm}$ channel. Live cell gating and quenching of extracellular signal were performed as described above.

To inhibit endocytosis by sHsp70 immobilisation, 96-well plates were coated with cmHsp70.1 or isotype control $(2 \mathrm{mg} / \mathrm{ml})$ in a $0.05 \mathrm{M}$ sodium carbonate buffer ( $\mathrm{pH} 9.6$ ), at $4{ }^{\circ} \mathrm{C}$ overnight. $2 \mu \mathrm{m}$ beads coated with goat anti-mouse IgG (chemicell, Berlin, Germany) were loaded with cmHsp70.1 or isotype control ( $2 \mu \mathrm{g} / \mathrm{mg}$ bead). $150 \mu \mathrm{g}$ of beads were distributed per well and incubated for $1 \mathrm{~h}$ at $37^{\circ} \mathrm{C}$. To determine the endocytic activity, cells were incubated in $100 \mu \mathrm{l}$ of ice-cold medium containing $7.5 \mu \mathrm{M}$ FM4-64 (Sigma) for $5 \mathrm{~min}$ and endocytosis was initiated by releasing the cold block at $37^{\circ} \mathrm{C}$ for another $5 \mathrm{~min}$. Following extensive washing, FM4-64 was excited at 500/9 nm and the fluorescence signal was measured at $730 / 20 \mathrm{~nm}$ in a plate reader (Tecan Infinite Pro, Tecan, Maennedorf, Switzerland). Cell-free wells served as background control.

To study endocytosis in the presence of Hsp70 oligomerisation inhibitors, cells plated onto 96-well plates were induced to overexpress Hsp70 for $12 \mathrm{~h}$. Next, cells were incubated with Hsp70 peptide fragments generated as described in Aprile et al. [10] or with BSA $(10-1000 \mathrm{~nm})$ at $37^{\circ} \mathrm{C}$ for $16 \mathrm{~h}$. Then endocytosis of FM4-64 was measured as described above with the temperature block performed at room temperature $\left(20^{\circ} \mathrm{C}\right)$.

The Hsp70 surface distribution on cell surfaces was studied under physiological conditions by simultaneous topography and recognition imaging (TREC). TREC is based on atomic force microscopy in combination with a recognition molecule on the tip of the cantilever, which enables simultaneous sensing of topography and recognition of proteins with nanometre accuracy. TREC was performed on a commercially available AFM set-up, a PicoPlus AFM 5500 (Agilent Technologies Inc., Chandler, AZ, USA) in magnetic AC (MAC) mode equipped with a PicoTREC box (Agilent Technologies Inc., Chandler, AZ, USA). Here the magnetically coated AFM tips (MACLevers, Type VII, Agilent Technologies Inc., AZ, USA) were functionalized with an antibody directed against surface Hsp70, isotype control, or the K4 peptide complementary for the
E3 tag on transgenic Hsp70. See Supplementary material and methods for full details.

For high-speed atomic force microscopy measurements $1 \mu \mathrm{g} / \mathrm{ml}$ recombinant Hsp70 was incubated on a freshly cleaved mica surface in PBS buffer for 5 min followed by several rinsing steps to remove unattached molecules from the liquid cell. Supported lipid bilayers in PBS buffer supplemented with $10 \mathrm{mM} \mathrm{MgCl} 2$ were prepared from dioleoyl-phosphatidylcholine(PC)/octadecanoyl-sphingomyelin (SM)/cholesterol (65/25/10 molar ratio; Avanti Polar Lipids Inc., Alabaster, AL, USA) vesicles as described in [11].

Values in the text and graphs are presented as mean \pm S.D. $P$ values are indicated in the graphs and were considered statistically significant when $<0.05$.

For a complete list of materials and methods used in this study please refer to the Supplementary information.

\section{Results and discussion}

To determine whether Hsp70 expression levels generally have an impact on the endocytic activity in unstressed cells, we used stably transfected tetracycline-inducible systems for Hsp70 overexpression in mouse B16 and human A375 melanoma cells (Hsp70) [9]. Cells stably transfected with empty vector served as control (ctrl). We measured the activity of endocytic pathways through the uptake of the established fluorescent tracers FM4-64 for general endocytic activity, transferrin-AlexaFluor488 (Tf) for clathrin-dependent endocytosis (CDE) and BODIPYlactosylceramide (LacCer) for clathrin-independent endocytosis (CIE) [12]. Upon Hsp70 overexpression in B16 and A375 cells, uptake of LacCer was significantly increased, while Tf uptake was slightly reduced (Fig. 1A). The latter observation was somewhat surprising, as previous reports on hepatoblastoma cells showed enhanced $C D E$, yet upon heat shock or pharmacological Hsp70 induction [13]. In fact, at stress conditions Hsp70 has been proposed to be able to substitute for Hsc70 in clathrin coat disassembly during $\mathrm{CDE}$. In this current study, endocytosis has been measured at unstressed conditions, and a slight decrease in CDE may be attributed to excess of sHsp70 competing with Tf as a substrate of CDE. This explanation is supported by the fact that a large fraction of $\operatorname{sHsp70}(60 \%)$ is endocytosed via CDE (Fig. 1B). Importantly, B16 cells with low basal level of Hsp70 displayed a considerable increase $(+30.0 \%)$ in their general endocytosis levels, attributable to an increase in CIE. A smaller difference measured for A375 cells with high basal level of Hsp70 pointed to a saturating effect of overexpression (Fig. 1C). Given the higher response upon Hsp70 overexpression, B16 cells and the robust marker FM4-64 were used in further experiments.

As an attempt to specifically interfere with Hsp70-mediated endocytosis, we applied a specific antibody (cmHsp70.1) recognising the substrate-binding subdomain (SBSD) of sHsp70. Apparently, this treatment had no effect on endocytosis (Fig. 1D), indicating that substrate binding of Hsp70 may not be necessary for Hsp70-mediated endocytosis. Since the Hsp70-antibody adduct retains mobility of sHsp70 [9], we next targeted sHsp70 by anti-Hsp70 antibody (cmHsp70.1) immobilised on bead and well surfaces (see drawing in Fig. 1F). Importantly, this approach was specific to sHsp70 and revealed a sizeable facilitation of endocytosis by sHsp70 in Hsp70 overexpressing cells (44.5\% and 18.3\% inhibition with antibody-coated bead + well and bead surfaces, respectively). It is noted that no significant inhibition of endocytosis was detected for control, low Hsp70 expressing cells (data not shown). As soluble anti-Hsp70 antibody did not have any effect on FM4-64 uptake of Hsp70 overexpressing cells (Fig. 1D), we conclude that limiting Hsp70 mobility itself, and no other eventually allosteric effects induced by the antibody, reduced endocytic activity. 

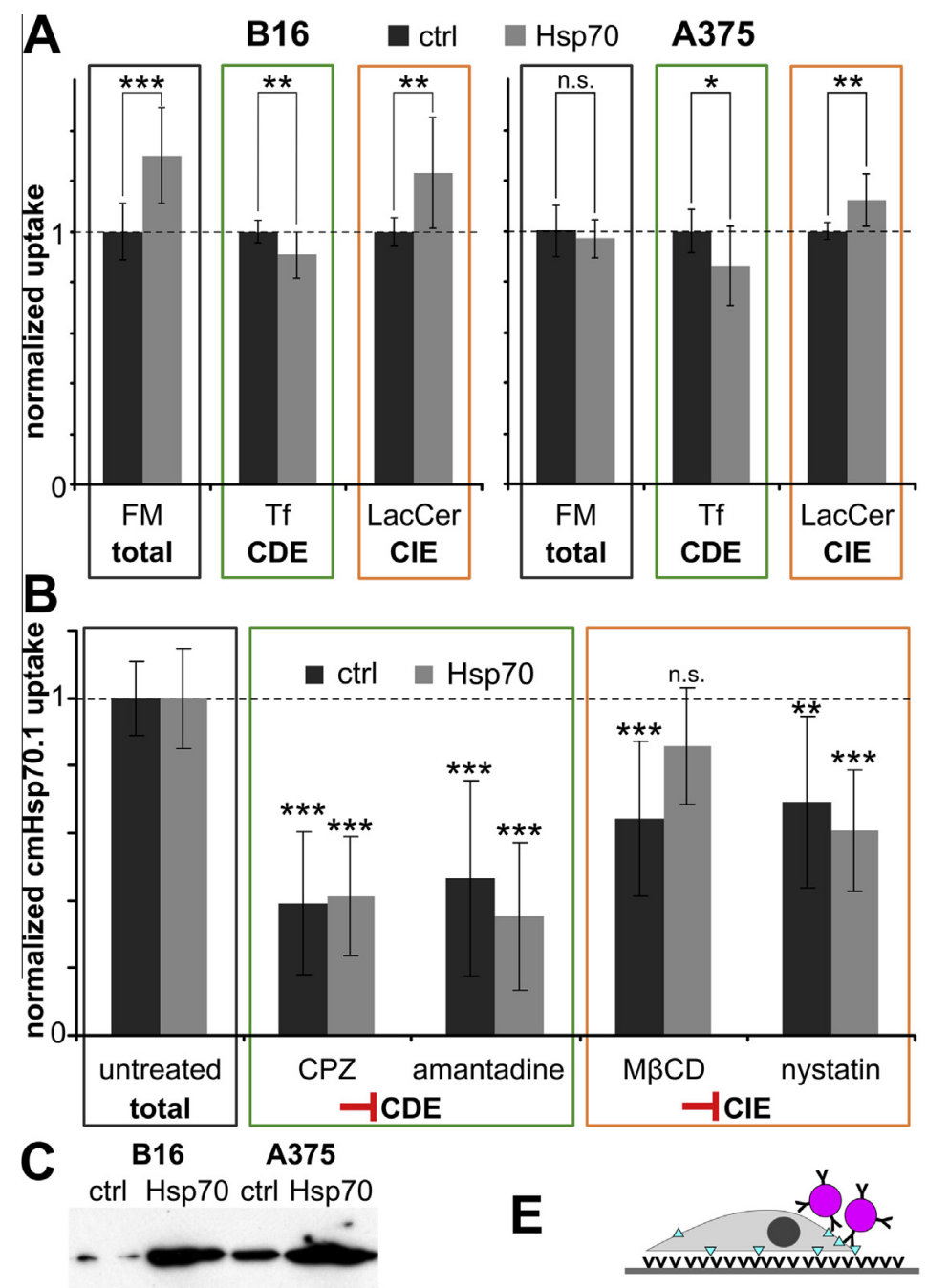

\section{B16 Hsp70}
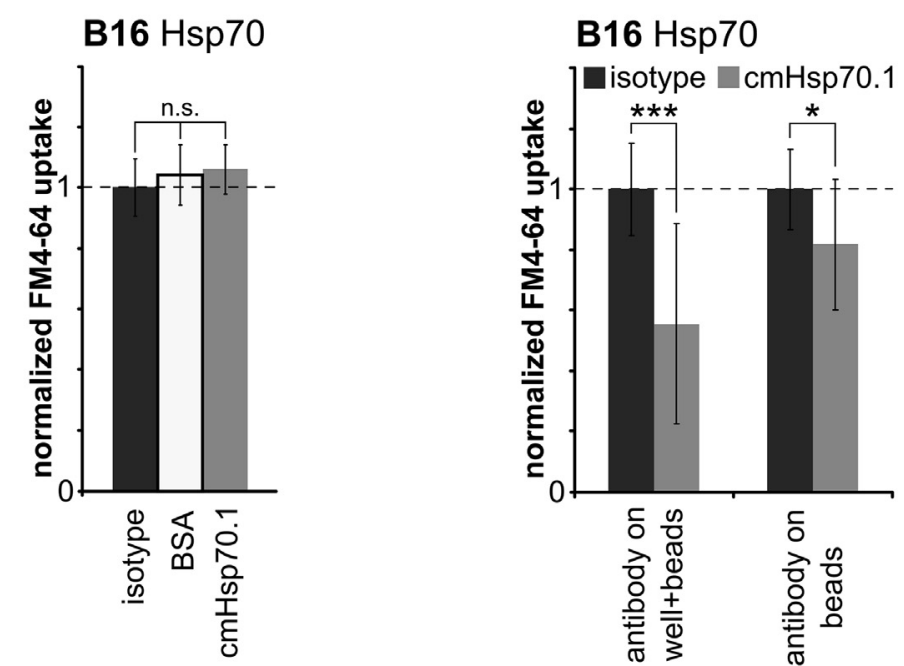

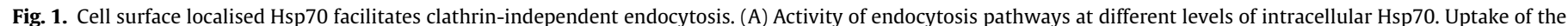

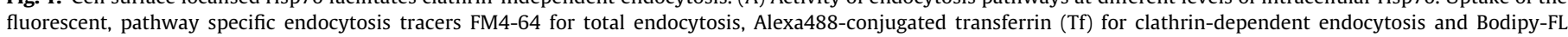

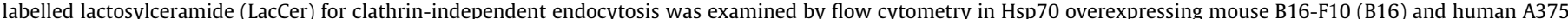

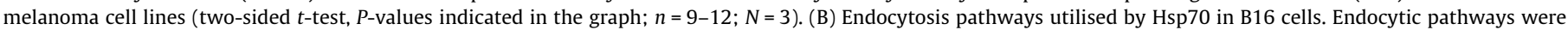

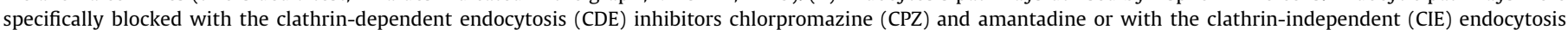

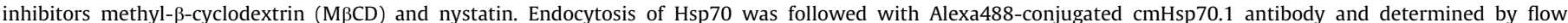

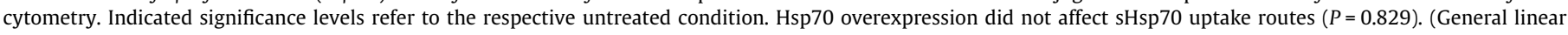

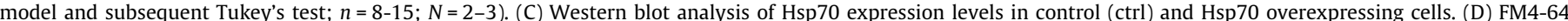

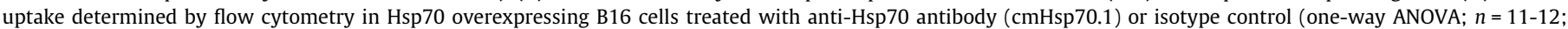

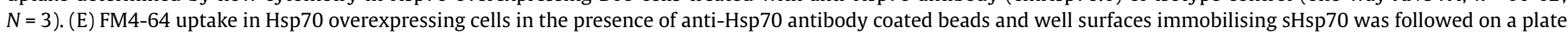
reader (one-sided $t$-test; $n=9-14 ; N=3$ ). 

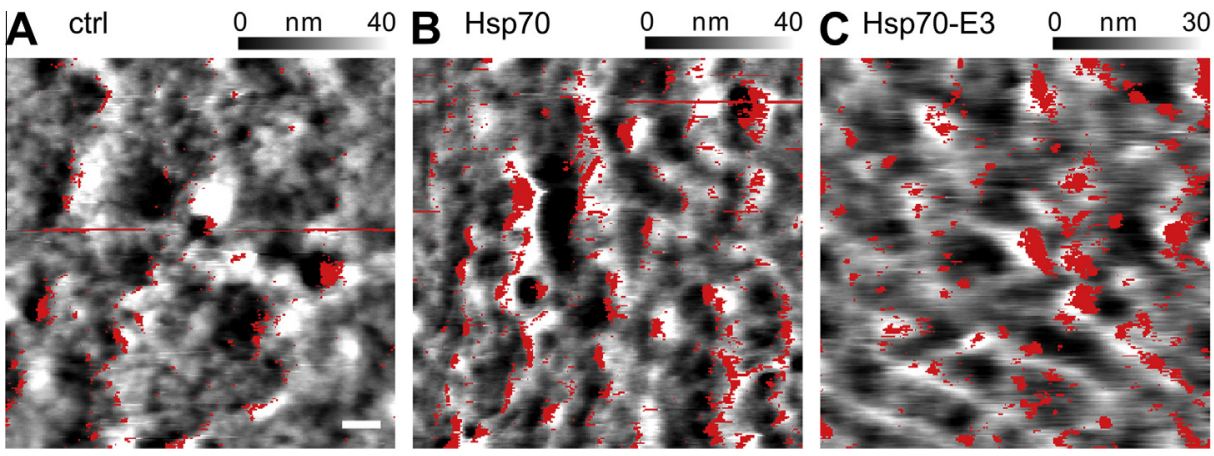

$30 \mathrm{D}$ cluster size distribution

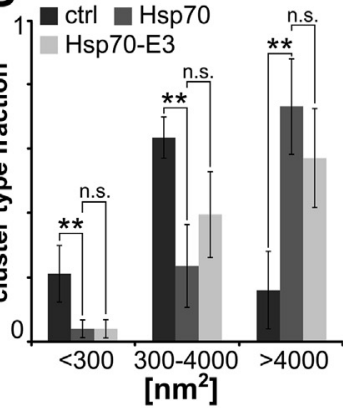

Fig. 2. Hsp70 accumulates in enlarged clusters in Hsp70 overexpressing B16 cell membranes. (A)-(C) Nano-organisation of Hsp70 on the cell surface. Control (ctrl), Hsp70 and Hsp70 conjugated to an E3 tag overexpressing cells were fixed and imaged by atomic force microscopy dynamic recognition imaging (TREC). Hsp70 was recognised by cmHsp70.1 antibody or E3-complement peptide K4 linked to the cantilever, respectively (red areas). The topography is displayed in grey, the scale bar is $200 \mathrm{~nm}$. (D) Relative Hsp70 cluster size distribution based on Hsp70 recognition images (one-way ANOVA and subsequent Tukey’s test; $n=3-4 ; N=3$ ).

The finding that mobility of sHsp70 was required for its effect on endocytosis indicated that stimulation of endocytosis required dynamic processes, possibly self-organisation of sHsp70 or interaction with other proteins. As clustering has been shown to facilitate endocytosis of diverse proteins such as LDL-receptors, amyloid precursor protein or $\beta_{1}$ integrin in both clathrin-dependent and -independent pathways [14-16], we hypothesized a similar mechanism for sHsp70-mediated endocytosis. To understand whether Hsp70 clusters exist on the cell surface and whether their size correlates with the effects of Hsp70 overexpression on endocytosis, we studied the nano-organisation of sHsp70 on ctrl, Hsp70 and E3 tagged Hsp70 overexpressing cells [17]. Using an atomic force
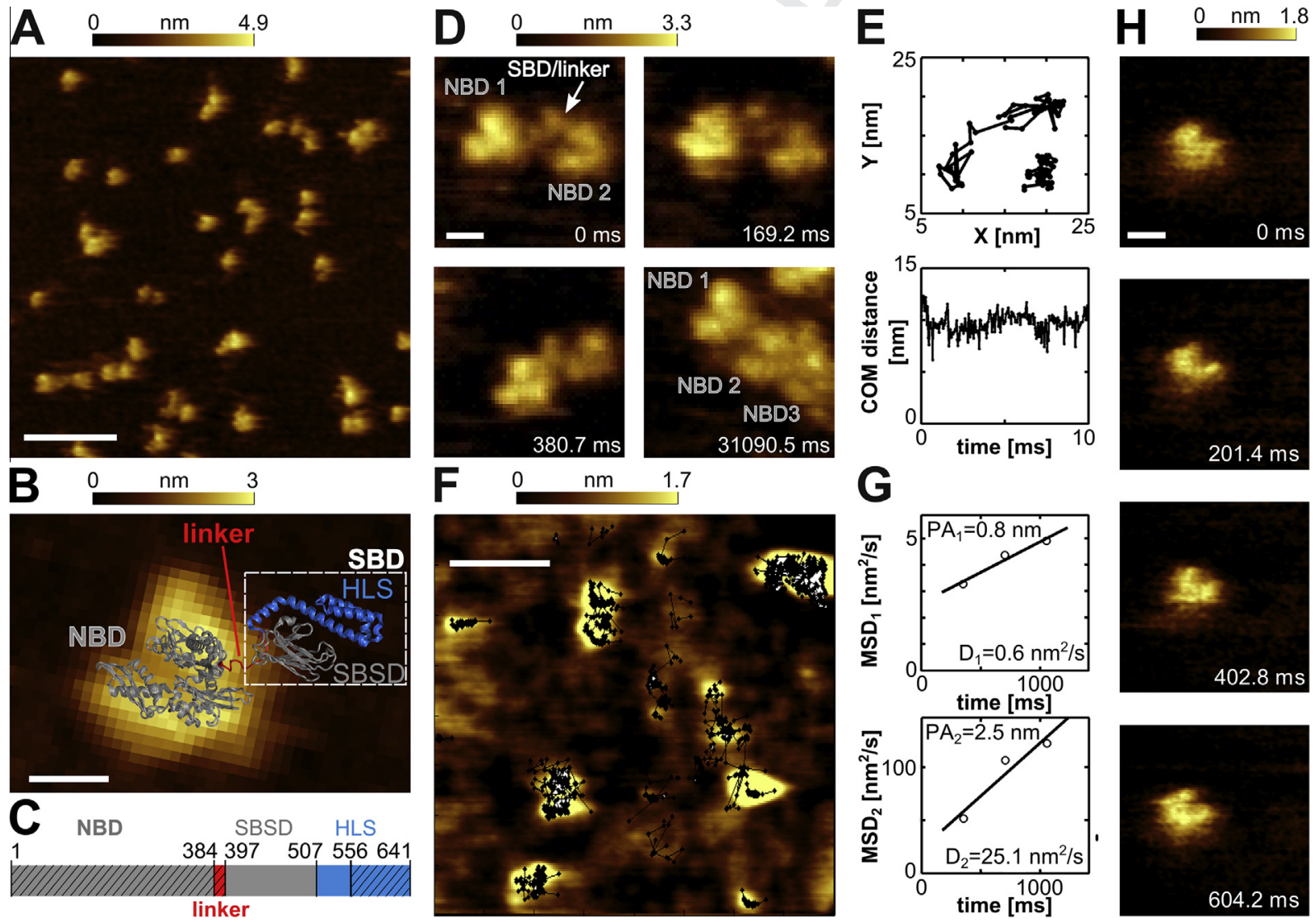

G
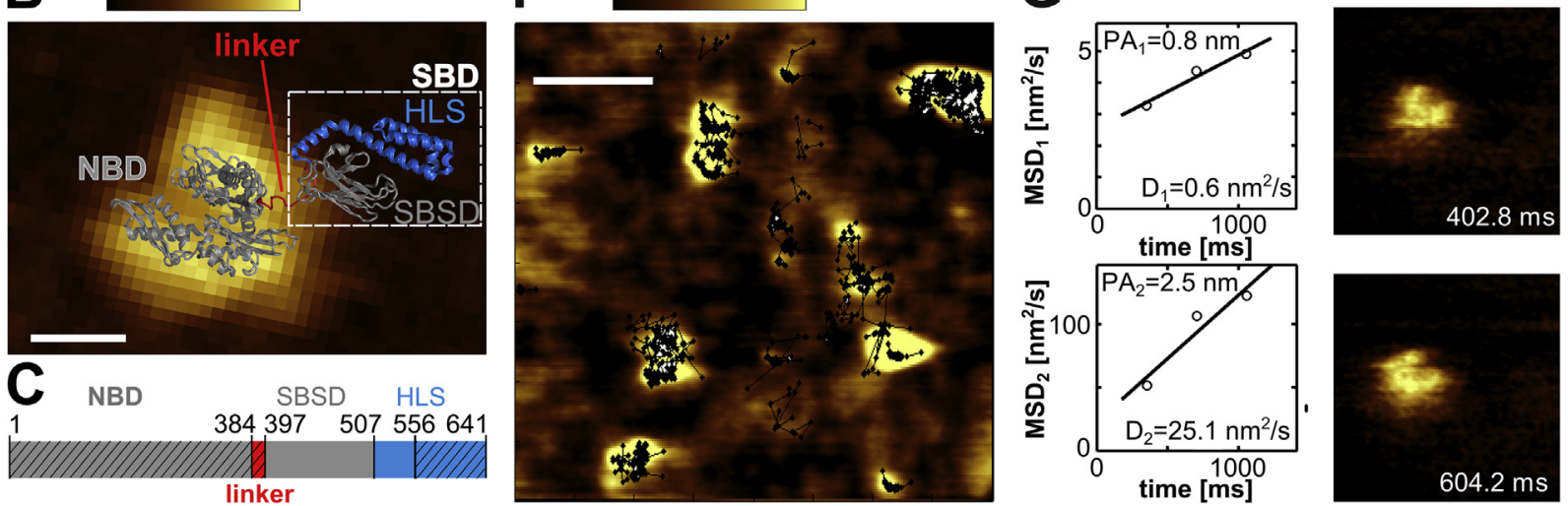

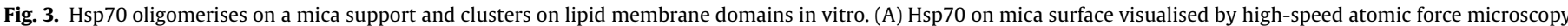

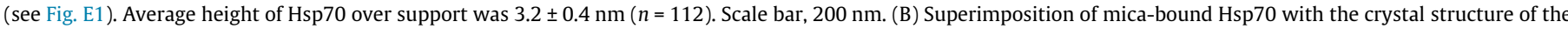

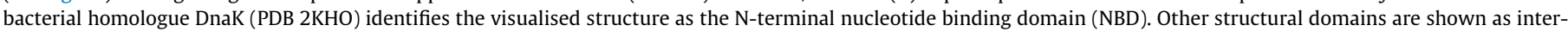

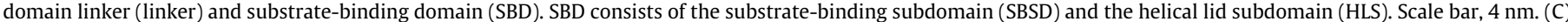

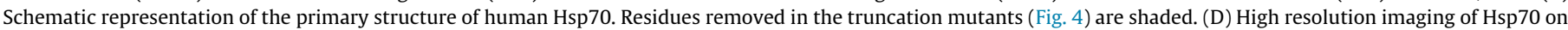

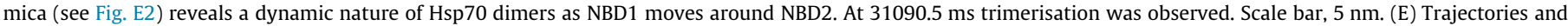

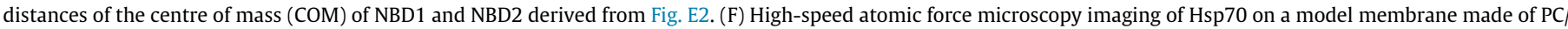

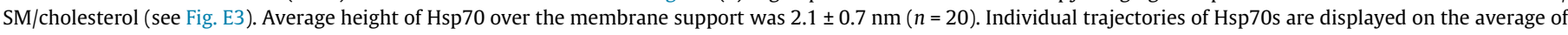

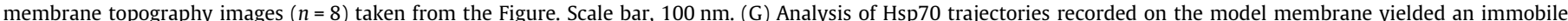

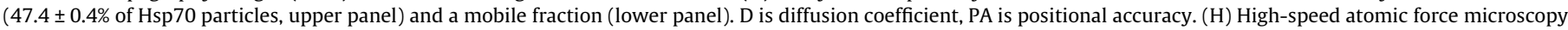
of Hsp70 on a PC/SM/cholesterol model membrane identifying the NBD as membrane anchoring domain (compare to Fig. 3B). Scale bar, 10 nm. 
microscopy cantilever conjugated to anti-Hsp70 antibody or to an E3 interacting K4 peptide, both topography and sHsp70 specific recognition images were recorded beyond optical resolution limits [18]. The Hsp70-E3/K4 system served as a control for the Hsp70/antibody system. As shown in Fig. 2, recognition maps (displayed in red) revealed that sHsp70 tended to accumulate in nano-domains (or clusters). In agreement with our hypothesis, a remarkable increase in cluster size from small $\left(\leqslant 300 \mathrm{~nm}^{2}\right.$, equivalent circular $\emptyset \sim 20 \mathrm{~nm})$ and medium $\left(>300 \mathrm{~nm}^{2},<4000 \mathrm{~nm}^{2}\right)$ to large clusters ( $\geqslant 4000 \mathrm{~nm}^{2}$, equivalent circular $\emptyset \sim 70 \mathrm{~nm}$ ) was observed as a result of Hsp70 overexpression. The larger cluster sizes of sHsp70 were also paralleled with a twofold increase in the surface density of Hsp70 on Hsp70 overexpressing cells compared to control (see Table E1), matching our previous data acquired by flow cytometry [9].

A possible factor that may facilitate clustering of sHsp70 is oligomerisation. In line with previous data obtained with biochemical measurements [10], dimers and higher order oligomers of recombinant human Hsp70 could be visualised by high resolution, high-speed atomic force microscopy at a liquid-solid interface [19]. Remarkably, Hsp70 monomers, dimers and higher oligomers all had a regular height of $3.2 \pm 0.4 \mathrm{~nm}$ indicating a uniform orientation on the support (Fig. 3A; Fig. E1). Analysing the recombinant protein sample at higher resolution, the nucleotide-binding domains (NBDs) of dimers and trimers could be clearly identified. The substrate-binding domains (SBDs) were poorly resolved likely due to their lack of association to the surface and their flexible connection to the NBD via the linker domain (Fig. 3B-D). Analysis of trajectories of NBDs of the same molecule revealed a fairly stable, yet flexible and dynamic dimer structure with an average distance of $9.7 \pm 0.9 \mathrm{~nm}$ between the NBDs (Fig. 3D and E; see Fig. E2). A dynamic formation of trimers was also evident (Fig. 3D).

The partial uptake of sHsp70 via the cholesterol-dependent CIE (Fig. 1B) and the affinity of Hsp70 to specific lipids such as cholesterol $[7,20]$, may further support accumulation and clustering of sHsp70 in cholesterol-enriched lipid rafts. To test this idea, interaction of recombinant Hsp70 with a supported bilayer was monitored by high-speed atomic force microscopy. A segregated lipid layer of $\mathrm{PC} / \mathrm{SM} /$ cholesterol was used as an artificial model, where lower non-raft and higher raft-like lipid domain areas were apparent [21]. Hsp70 added to the lipid layer essentially localised and clustered on the elevated, raft like domains (Fig. 3F). With nearly equal probability, a practically immobile and a mobile fraction of Hsp70 could be detected in all lipid domains analysed (Fig. 3G, see Fig. E3). Notably, the reduced average height of Hsp70 on the lipid bilayer $(2.1 \pm 0.7 \mathrm{~nm})$, as compared to impenetrable mica $(3.2 \pm 0.4 \mathrm{~nm})$, indicated a partial insertion of Hsp70 into the lipid layer (Fig. $3 \mathrm{~A}$ and $\mathrm{F} ; \quad P<0.001$ ). Furthermore, time-resolved high-resolution images revealed the NBD as the lipid interaction site (Fig. $3 \mathrm{H}$ ). This finding was in good agreement with previous reports on a single amino acid mutation in the NBD that impaired Hsp70 membrane interaction [22,23]. Taken together, these data showed that Hsp70 was able to interact with artificial lipid membranes via its NBD domain and accumulated in raft-like lipid domains, where it oligomerised and formed clusters.

Finally, to test if Hsp70 self-organisation stimulates endocytosis, we used fragments of the recombinant human Hsp70 with impaired oligomerisation properties, hence capable of interfering with the oligomerisation of $w t$ full length Hsp70. It has been recently proposed that Hsp70 oligomerisation is mediated by the
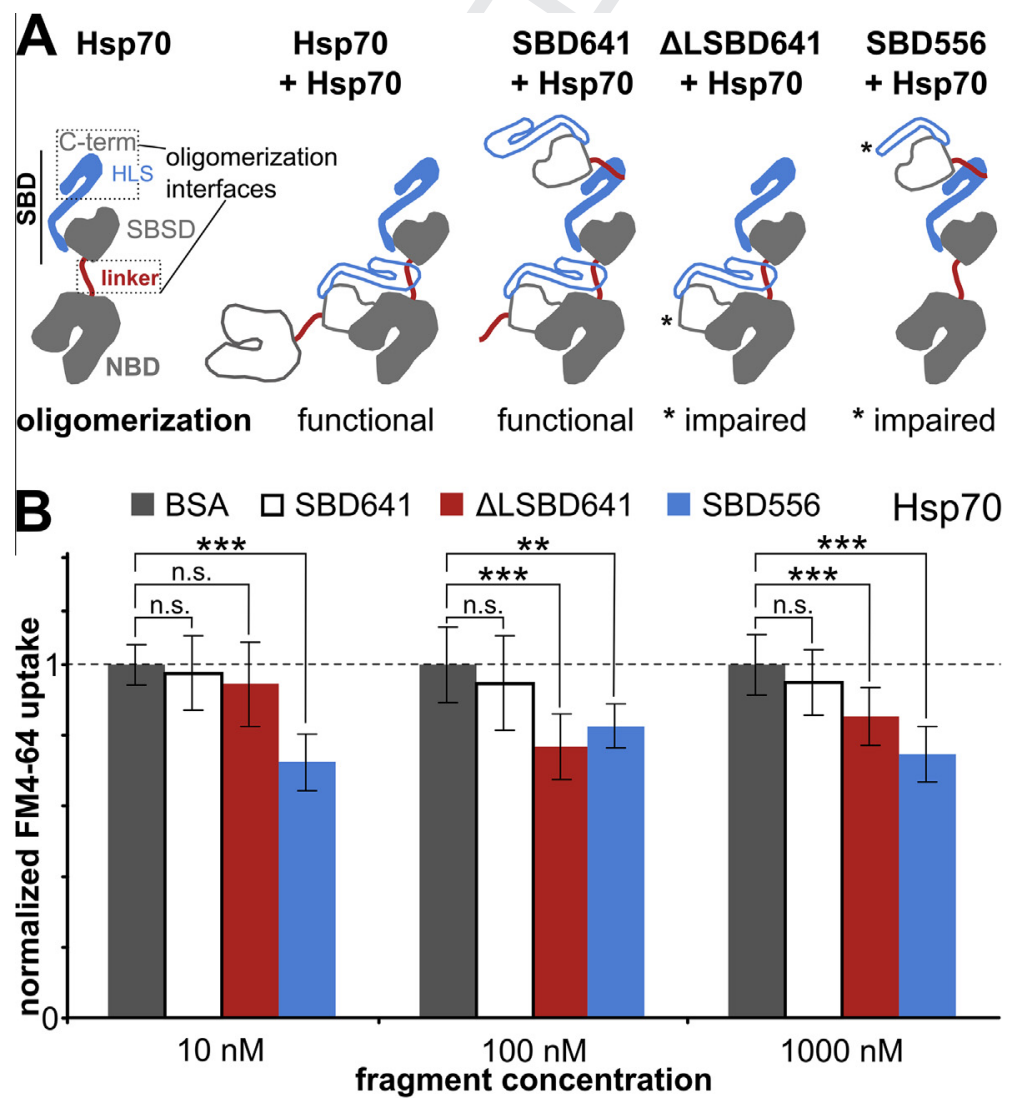

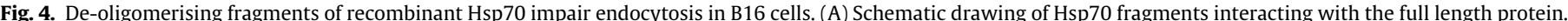

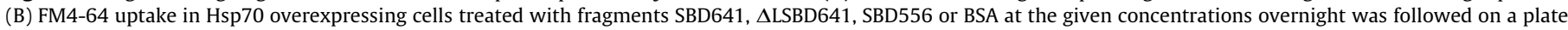
reader (one-way ANOVA and subsequent Tukey's test; $10 \mathrm{~nm} n=17-12, N=5 ; 100 \mathrm{~nm} n=8-9, N=2 ; 1000 \mathrm{~nm} n=17-23, N=5$ ). 


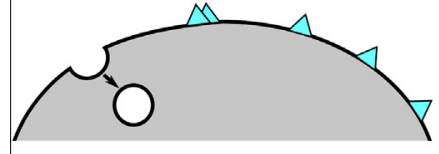

ctrl

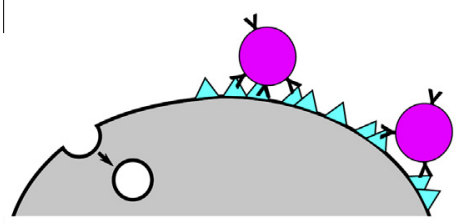

Hsp70: immobilised

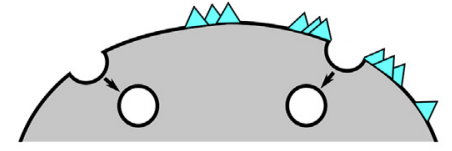

Hsp70

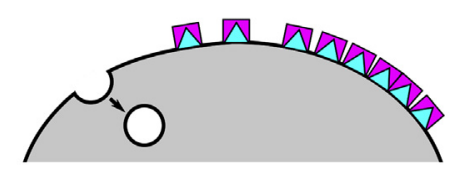

Hsp70: de-oligomerised

Fig. 5. Schematic model for surface localised Hsp70-mediated facilitation of endocytosis in cancer cells. In control cells with low level of Hsp70, tumour cell surface localised Hsp70 (sHsp70) is localised in smaller size clusters on the plasma membrane. Upregulating intracellular Hsp70 expression, as in various forms of cancer [2], is accompanied with an elevated sHsp70 concentration [9]. sHsp70 at higher concentration forms larger clusters on the plasma membrane, which enables facilitation of CIE. Immobilisation or de-oligomerisation of sHsp70 interfere with sHsp70-mediated facilitation of endocytosis, indicating that dynamics of sHsp70 as well as its ability to oligomerise are important to exert an effect on endocytosis.

inter-domain linker (linker), connecting the NBD and the SBD, and the C-terminal part of the helical lid subdomain (HLS) of the SBD [10]. By adding SBD fragments of recombinant human Hsp70 lacking either of the two oligomerisation interfaces ( $\triangle$ LSBD641 variant without the linker and SBD556 variant without the C-terminal part of the HLS; see Figs. $3 \mathrm{C}$ and $4 \mathrm{~A}$ ) to Hsp70 overexpressing cells, we expected to reduce sHsp70 oligomerisation, hence endocytosis. As these fragments lacked the NBD domain, we thereby eliminated potential binding of the fragments to the cell membrane (Fig. $3 \mathrm{H}$ ). Indeed, interfering with oligomerisation of sHsp70 by the SBD556 and $\triangle$ LSBD641 fragments significantly reduced the FM4-64 uptake in a concentration dependent manner (Fig. 4B, up to $27.7 \%$ inhibition). In contrast, neither inert BSA nor a control fragment with the two fully functional oligomerising interfaces, did affect endocytosis at any concentration tested. It should be noted that administering with $w t$ full length recombinant Hsp70 slightly increased FM4-64 uptake (data not shown). It is also noteworthy that extent of the observed inhibition was comparable to the stimulation of endocytosis by Hsp70 overexpression (Fig. 1A), supporting the idea of an oligomerisation dependent mechanism.

In conclusion, we propose a novel function of the cell surface localised Hsp70 as a stimulator of CIE. Accumulation of overexpressed Hsp70 in large size nano-domains on the cell surface, paralleled with a reversible increase in endocytosis driven by sHsp70 oligomerisation, strongly suggest a clustering dependent mechanism for sHsp70-mediated increase in endocytosis (Fig. 5). Interestingly, oligomerisation was previously described as a requirement for raft-association and transcytosis of GPI-GFP $[24,25]$ as well as for the recognition and internalisation of toxic amylin [26]. Here we first describe sHsp70 as a potential regulator of such oligomerisation and clustering dependent endocytic events. Preferential interaction of Hsp70 with specific lipids, its ability to cluster on cell membranes and its chaperone function support a significant role for sHsp70 in endocytosis. In line with this argument, clustering and interaction of specific surface molecules with lipid rafts are thought to enable formation of membrane curvature and invagination, hence initiating endocytosis even without adaptor proteins at the cytosolic side $[27,28]$. We suggest that sHsp70, when present in cancer cell membranes, may facilitate the raft associated CIE in an analogous manner. Revealing additional components of the CIE machinery and further understanding of the mechanism of action of known regulators, such as glycosphingolipids, Galectin-3 or sHsp70 will be fostering this emerging field [27].

Altered endocytosis is a typical feature of cancer, where dynamic remodelling and recycling routes at the plasma membrane support tumour cell survival and progression [8]. As Hsp70 is frequently upregulated in tumours, the resulting increased sHsp70 levels $[4,9]$ could stimulate endocytosis for the benefit of the tumour $[29,30]$. Inhibition of the sHsp70-mediated stimulation of endocytosis, as shown in this study, may therefore represent an adjuvant therapeutic strategy against cancer. At the same time, tumour specific surface localisation of Hsp70 allows specific drug targeting of cancer cells [31,32], where sHsp70-mediated increase in endocytosis would enhance the efficiency of drug treatment. sHsp70-mediated stimulation of endocytosis, reported in this paper, represents a novel cancer specific mechanism and further validates the tumour marker sHsp70 for efficient anti-cancer drug delivery. In future, we aim to follow our current working hypothesis in order to characterise the mechanism of regulation of endocytosis by sHsp70.

\section{Competing interest}

The authors declare no competing interests.

\section{Acknowledgments}

We thank Prof. Hermann J. Gruber (Institute for Biophysics, Johannes Kepler University, Linz, Austria) for developing the atomic force microscopy tip chemistry protocol for the NHS-PEG-maleimide linker. Prof. Boris Margulis (Institute of Cytology, Russian Academy of Sciences, St. Petersburg, Russia) kindly provided us with full length recombinant Hsp70.

This work was funded by the European Regional Development Fund (EFRE), the state of Upper Austria. Laszlo Vigh was supported by the Hungarian Basic Research Fund (OTKA, No. 100857). Nunilo Cremades is a Royal Society Dorothy Hodgkin Research Fellow.

\section{Appendix A. Supplementary data}

Supplementary data associated with this article can be found, in the online version, at http://dx.doi.org/10.1016/j.febslet.2015.07. 037.

\section{References}

[1] Mayer, M.P. (2013) Hsp70 chaperone dynamics and molecular mechanism. Trends Biochem. Sci. 38, 507-514.

[2] Calderwood, S.K., Khaleque, M.A., Sawyer, D.B. and Ciocca, D.R. (2006) Heat shock proteins in cancer: chaperones of tumorigenesis. Trends Biochem. Sci. 31, 164-172. 
[3] Juhasz, K., Lipp, A.-M., Nimmervoll, B., Sonnleitner, A., Hesse, J., Haselgruebler, T. and Balogi, Z. (2013) The complex function of Hsp70 in metastatic cancer. Cancers 6, 42-66.

[4] Hantschel, M., Pfister, K., Jordan, A., Scholz, R., Andreesen, R., Schmitz, G. Schmetzer, H., Hiddemann, W. and Multhoff, G. (2000) Hsp70 plasma membrane expression on primary tumor biopsy material and bone marrow of leukemic patients. Cell Stress Chaperones 5, 438-442.

[5] Multhoff, G., Botzler, C., Jennen, L., Schmidt, J., Ellwart, J. and Issels, R. (1997) Heat shock protein 72 on tumor cells: a recognition structure for natural killer cells. J. Immunol. 158, 4341-4350.

[6] Stangl, S., Gehrmann, M., Riegger, J., Kuhs, K., Riederer, I., Sievert, W., Hube, K., Mocikat, R., Dressel, R., Kremmer, E., Pockley, A.G., Friedrich, L., Vigh, L., Skerra, A. and Multhoff, G. (2011) Targeting membrane heat-shock protein 70 (Hsp70) on tumors by cmHsp70.1 antibody. Proc. Natl. Acad. Sci. 108, 733-738.

[7] Vega, V.L., Rodriguez-Silva, M., Frey, T., Gehrmann, M., Diaz, J.C., Steinem, C., Multhoff, G., Arispe, N. and De Maio, A. (2008) Hsp70 translocates into the plasma membrane after stress and is released into the extracellular environment in a membrane-associated form that activates macrophages. J. Immunol. 180, 4299-4307.

[8] Mosesson, Y., Mills, G.B. and Yarden, Y. (2008) Derailed endocytosis: an emerging feature of cancer. Nat. Rev. Cancer 8, 835-850.

[9] Juhasz, K., Thuenauer, R., Spachinger, A., Duda, E., Horvath, I., Vigh, L., Sonnleitner, A. and Balogi, Z. (2013) Lysosomal rerouting of Hsp70 trafficking as a potential immune activating tool for targeting melanoma. Curr. Pharm. Des. 19, 430.

[10] Aprile, F.A., Dhulesia, A., Stengel, F., Roodveldt, C., Benesch, J.L.P., Tortora, P., Robinson, C.V., Salvatella, X., Dobson, C.M. and Cremades, N. (2013) Hsp70 Oligomerization Is Mediated by an Interaction between the Interdomain Linker and the Substrate-Binding Domain. PLoS One 8, e67961.

[11] Yamamoto, D., Uchihashi, T., Kodera, N., Yamashita, H., Nishikori, S., Ogura, T., Shibata, M. and Ando, T. (2010) High-speed atomic force microscopy techniques for observing dynamic biomolecular processesMethods in Enzymology, pp. 541-564, Elsevier.

[12] Sharma, D.K., Choudhury, A., Singh, R.D., Wheatley, C.L., Marks, D.L. and Pagano, R.E. (2003) Glycosphingolipids internalized via caveolar-related endocytosis rapidly merge with the clathrin pathway in early endosomes and form microdomains for recycling. J. Biol. Chem. 278, 7564-7572.

[13] Vega, V.L., Charles, W. and De Maio, A. (2010) A new feature of the stress response: increase in endocytosis mediated by Hsp70. Cell Stress Chaperones $15,517-527$.

[14] Heuser, J.E. and Anderson, R.G. (1989) Hypertonic media inhibit receptormediated endocytosis by blocking clathrin-coated pit formation. J. Cell Biol. $108,389-400$.

[15] Sharma, D.K., Brown, J.C., Cheng, Z., Holicky, E.L., Marks, D.L. and Pagano, R.E. (2005) The glycosphingolipid, lactosylceramide, regulates $\beta 1$-integrin clustering and endocytosis. Cancer Res. 65, 8233-8241.

[16] Schneider, A., Rajendran, L., Honsho, M., Gralle, M., Donnert, G., Wouters, F Hell, S.W. and Simons, M. (2008) Flotillin-dependent clustering of the amyloid precursor protein regulates its endocytosis and amyloidogenic processing in neurons. J. Neurosci. 28, 2874-2882.

[17] Yano, Y., Yano, A., Oishi, S., Sugimoto, Y., Tsujimoto, G., Fujii, N. and Matsuzaki, K. (2008) Coiled-coil tag - probe system for quick labeling of membrane receptors in living cell. ACS Chem. Biol. 3, 341-345.
[18] Chtcheglova, L.A., Waschke, J., Wildling, L., Drenckhahn, D. and Hinterdorfer, P. (2007) Nano-scale dynamic recognition imaging on vascular endothelial cells. Biophys. J. 93, L11-L13.

[19] Preiner, J., Kodera, N., Tang, J., Ebner, A., Brameshuber, M., Blaas, D., Gelbmann, N., Gruber, H.J., Ando, T. and Hinterdorfer, P. (2014) IgGs are made for walking on bacterial and viral surfaces. Nat. Commun. 5.

[20] Gehrmann, M., Liebisch, G., Schmitz, G., Anderson, R., Steinem, C., De Maio, A., Pockley, G. and Multhoff, G. (2008) Tumor-specific Hsp70 plasma membrane localization is enabled by the glycosphingolipid Gb3. PLoS One 3, e1925.

[21] Sullan, R.M.A., Li, J.K., Hao, C., Walker, G.C. and Zou, S. (2010) Cholesteroldependent nanomechanical stability of phase-segregated multicomponent lipid bilayers. Biophys. J. 99, 507-516.

[22] Kirkegaard, T., Roth, A.G., Petersen, N.H.T., Mahalka, A.K., Olsen, O.D., Moilanen, I., Zylicz, A., Knudsen, J., Sandhoff, K., Arenz, C., et al. (2010) Hsp70 stabilizes lysosomes and reverts Niemann-Pick disease-associated lysosomal pathology. Nature 463, 549-553.

[23] Mahalka, A.K., Kirkegaard, T., Jukola, L.T.I., Jäättelä, M. and Kinnunen, P.K.J. (2014) Human heat shock protein 70 (Hsp70) as a peripheral membrane protein. Biochim. Biophys. Acta 1, 1-10.

[24] Brameshuber, M., Weghuber, J., Ruprecht, V., Gombos, I., Horvath, I., Vigh, L., Eckerstorfer, P., Kiss, E., Stockinger, H. and Schütz, G.J. (2010) Imaging of mobile long-lived nanoplatforms in the live cell plasma membrane. J. Biol. Chem. 285, 41765-41771.

[25] Galmes, R., Delaunay, J.-L., Maurice, M. and Aït-Slimane, T. (2013) Oligomerization is required for normal endocytosis/transcytosis of a GPIanchored protein in polarized hepatic cells. J. Cell Sci. 126, 3409-3416.

[26] Trikha, S. and Jeremic, A.M. (2011) Clustering and internalization of toxic amylin oligomers in pancreatic cells require plasma membrane cholesterol. J. Biol. Chem. 286, 36086-36097.

[27] Lakshminarayan, R., Wunder, C., Becken, U., Howes, M.T., Benzing, C., Arumugam, S., Sales, S., Ariotti, N., Chambon, V., Lamaze, C., Loew, D., Shevchenko, A., Gaus, K., Parton, R.G. and Johannes, L. (2014) Galectin-3 drives glycosphingolipid-dependent biogenesis of clathrin-independent carriers. Nat. Cell Biol. 16, 595-606.

[28] Romer, W., Berland, L., Chambon, V., Gaus, K., Windschiegl, B., Tenza, D., Aly, M.R., Fraisier, V., Florent, J.C., Perrais, D., Lamaze, C., Raposo, G., Steinem, C., Sens, P., Bassereau, P. and Johannes, L. (2007) Shiga toxin induces tubular membrane invaginations for its uptake into cells. Nature 450, 670-675.

[29] Joffre, C., Barrow, R., Ménard, L., Calleja, V., Hart, I.R. and Kermorgant, S. (2011) A direct role for Met endocytosis in tumorigenesis. Nat. Cell Biol. 13, 827-837.

[30] Sigismund, S., Woelk, T., Puri, C., Maspero, E., Tacchetti, C., Transidico, P., Di Fiore, P.P. and Polo, S. (2005) Clathrin-independent endocytosis of ubiquitinated cargos. Proc. Natl. Acad. Sci. U.S.A. 102, 2760-2765.

[31] Gaca, S., Reichert, S., Multhoff, G., Wacker, M., Hehlgans, S., Botzler, C., Gehrmann, M., Rödel, C., Kreuter, J. and Rödel, F. (2013) Targeting by cmHsp70.1-antibody coated and survivin miRNA plasmid loaded nanoparticles to radiosensitize glioblastoma cells. J. Control. Release Off. J. Control. Release Soc. 172, 201-206.

[32] Stangl, S., Gehrmann, M., Dressel, R., Alves, F., Dullin, C., Themelis, G., Ntziachristos, V., Staeblein, E., Walch, A., Winkelmann, I. and Multhoff, G. (2011) In vivo imaging of CT26 mouse tumours by using cmHsp70.1 monoclonal antibody. J. Cell Mol. Med. 15 (4), 874-887. 